\section{§11. Bend Points of Hydrogen Partial Pressure Curves Obtained by Tritium Removal Simulation Tests}

Kawano, T.

In previous studies, a conceptual tritium cleanup system was developed to remove tritium in various chemical forms from exhaust gases. This cleanup system differs from conventional ones employed at many facilities where tritium is handled in that tritium is removed as tritiated hydrogen molecules. In conventional systems, the tritium is removed in the form of water vapor.

The previously reported tritium cleanup system is shown schematically in Fig. 1. The system consists of five main components: hydrogen separator, decomposition processing vessel, hydrogen-absorbing vessel, circular pump, and buffer tank. The decomposition-processing vessel of the five main components was developed for this system in our laboratory.

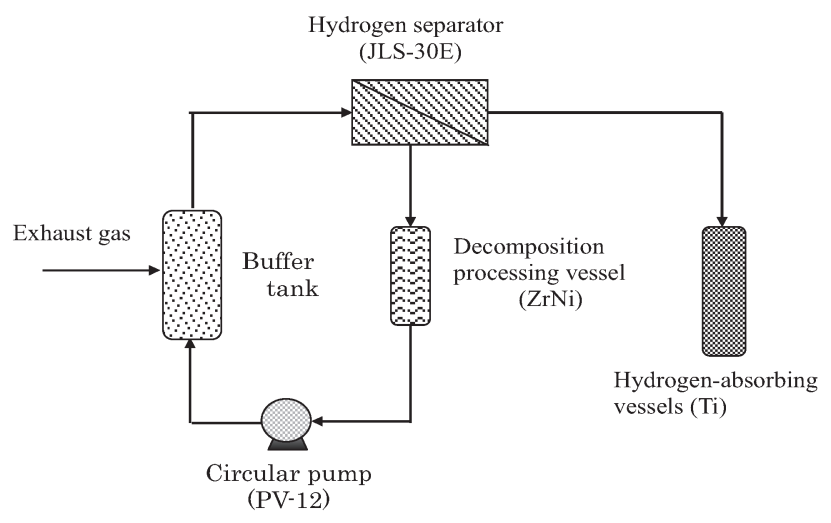

Fig. 1 Tritium-cleanup system containing a decomposition-processing vessel.

For the present study, it was assumed that the gas for processing was composed of hydrogen, methane, and helium. Some of the hydrogen and methane molecules were assumed to be tritiated, and helium was added to maintain a steady flow of gas through the stages of processing in the cleanup system. The performance of the tritium cleanup system was examined by computer-based simulation with simulated exhaust gas. To monitor removal of tritium from the exhaust gas, the partial pressures of hydrogen and methane were examined in a series of performance tests by computer simulation.

Results indicate that the partial pressure of hydrogen underwent a characteristic change with two bend points, even though the partial pressure of methane continued to decrease steadily. The time positions of the bends and time interval between the two bend points on the curve of hydrogen partial pressure were investigated under various conditions in relation to component fraction and gas volume.

Figure 2 shows results of these simulation tests and studies. In Fig. 2, the first and second bends and their time interval were plotted as a function of methane proportion for process gas volumes of $1,2,3$, and $6 \mathrm{Nm}^{3}$. Hydrogen proportions can be calculated by 0.95 minus methane proportion, where the proportion of 0.05 is attributed to helium. The y-axis represents elapsed time when the bends appear. In Fig. 2, open and closed symbols indicate up-curved bends and down-curved bends, respectively. Change of the first bend, the second bend and the time interval between the first and second bends are plotted in Fig. 2(A), 2(B) and 2(C).

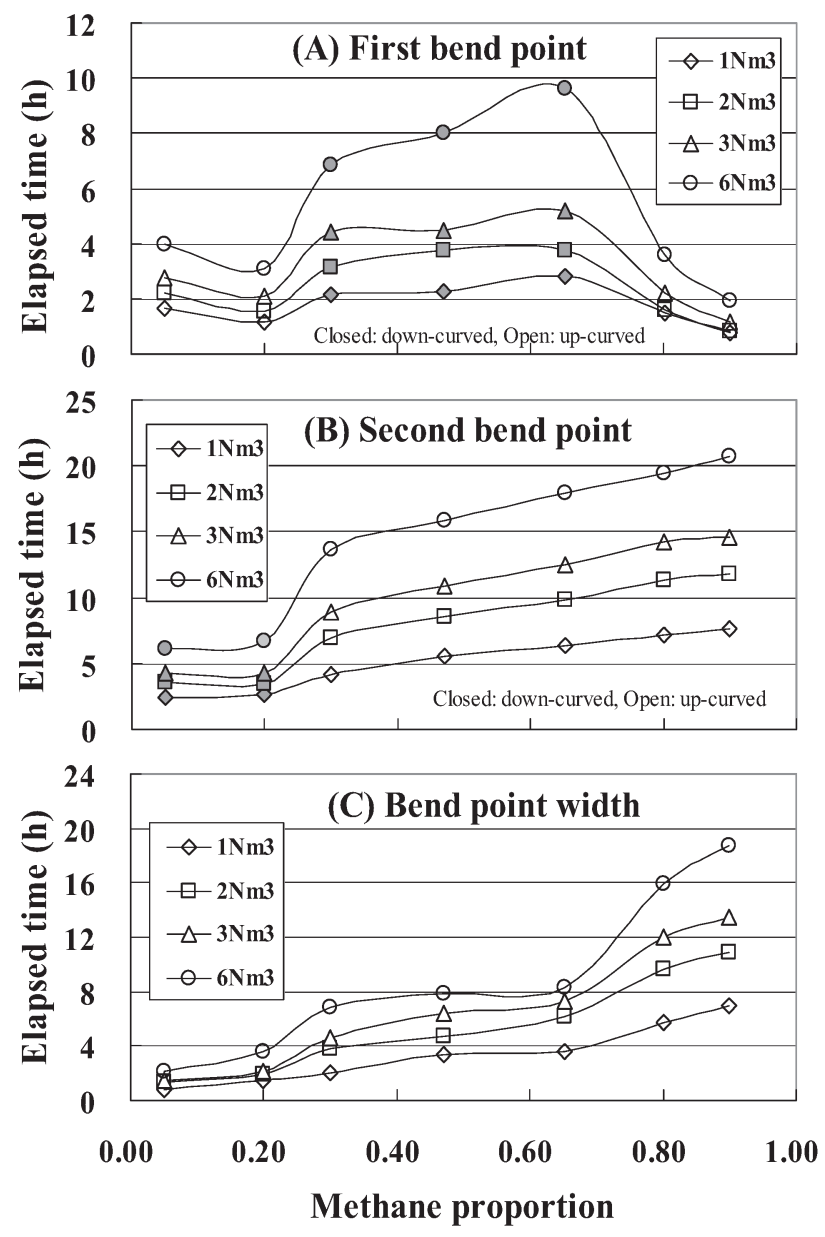

Fig. 2 Dependence of the first and second bends and their time interval on component proportion and gas volume to be processed.

The first bend appears to correspond to the completion of one cycle of processing and the second bend may correspond to the period where helium becomes the dominant determinant of pump speed. Both bends were observed at longer times, and the time interval between both bends increased as the amount of gas to be processed increased. Similarly, the second bend was observed at longer times, and the time interval between both bends increased with methane percentage. The results suggest that some features of exhaust gas can be estimated by inspecting both bend points, because the positions of the bend points and the time interval between them provided information about exhaust gas components and volume. 